\title{
Robust Reliability Optimal Design Based on Thermo-mechanical Coupling Compliant Mechanism
}

\author{
Cuiqin WU, Yinfeng WU \\ School of Mechanical and Electrical Engineering, Jiangxi University of Science and Technology, \\ Ganzhou, Jiangxi, China \\ cuiqinwu@163.com
}

Keywords: Thermo-mechanical coupling; Compliant mechanism; Topology optimization; Robust Optimal design

\begin{abstract}
Random variable of thermo-mechanical coupling (TMC) structure is analyzed first, then the topology optimization model of TMC compliant mechanism is established. According to the uncertainty factors such as temperature, load, volume ratio and thermal expansion coefficient in the process of topology optimization, the robust reliability optimal design method is introduced into the topology optimization process. The various factors on the sensitivity of topology optimization process is analyzed, the best match between various factors is find out, which make the product has robust reliability for the change of the uncertainty. The residual analysis for the most sensitive factors is carried out. Finally the correctness and validity of the method is verified with an example.
\end{abstract}

\section{Introduction}

When we study the compliant mechanisms by adopting topological optimization method, we only need to give design domain and allocate input and output position, no need from the perspective of a known rigidity. The mechanism come from this method have a input and output relationship of optimized “force-displacement", thus it attracts people's attention ${ }^{[1]}$. Topology optimization research is developing from certain topology optimization direction ${ }^{[2]}$ to reliable topology optimization direction ${ }^{[3]}$. Jinqing $\mathrm{Zhan}^{[4]}$ proposed a reliable topology optimization method of compliant mechanism based on infrastructure method. KHARMANDA ${ }^{[5]}$ proposed a reliable topology optimization method. Zhaokun $\mathrm{Li}^{[6]}$ proposed a geometric nonlinear topology optimization research method based on reliable compliant mechanism. Their research turned out that the mechanism based on reliable topology optimization is more reasonable than the mechanism based on certain topology optimization. Yongcong Kuang ${ }^{[7]}$ build a level set function to design variable, stiffness minimization, and proposed corresponding optimization algorithm. Mingtao Cui ${ }^{[8]}$ established a monolithic compliant mechanism multi-target reliable topology optimization design dual mathematical model.

This paper combines topology optimization process of compliant mechanism and robust reliability optimal design, analyzes the sensitivity of uncertainty random variables such as design domain's size, external load and temperature in topology optimization process. Then get the best combination among these random variables.

\section{Random Variable Coupling Field Analysis}

Hot objects will produce thermal expansion, thus caused by the thermal deformation and displacement of combined action of mechanical load and temperature load structure called a hot solid coupling structure ${ }^{[9]}$, the finite element balance equation ${ }^{[10]}$ :

$$
K U=F_{m}+F_{h}=F_{m}+\sum_{e=1}^{N} \varphi\left(\rho_{e}\right) \frac{\alpha E \Delta T}{2(1-v)} \cdot\left[\begin{array}{llllllll}
-1 & -1 & 1 & -1 & 1 & 1 & -1 & 1
\end{array}\right]^{T} .
$$

Where, $K$ is the whole stiffness matrix, $U$ is the displacement of the column, $F_{m}$ is the outside mechanical load,$F_{h}$ is the change of heat load caused by temperature, $\varphi\left(\rho_{e}\right)$ is the function of related to material properties, $\rho_{e}$ is the unit relative density design variables, $\alpha$ is thermal expansion 
coefficient, $E$ is the modulus of elasticity, $\Delta T$ is the change of temperature, $v$ is the Poisson's ratio, $N$ is the total number of nodes design domain.

when the temperature of the material properties and geometric size random variation, then the formula (1) would be rewritten:

$$
K(x) U(y)=F_{m}(x)+F_{h}(x) .
$$

Where, the $x$ is made up of $\mathrm{n}$ random parameters are random vector, $x=\left(x_{1}, x_{2}, \ldots, x_{\mathrm{n}}\right)^{T}$.

\section{The Robust Reliability Optimization Design}

The robust reliability optimal design by selecting the level of combination of controllable factors to reduce the sensitivity of the product or process changes for noise, thus achieve the purpose of reducing the product or process performance fluctuations.

Analysis Of Noise Factors in the Topology Optimization of TMC Compliant Mechanism. Noise factors are factors that cannot be easily controlled in normal production process or service conditions. The noise factors in the topology optimization process of TMC compliant mechanism including temperature, geometry size, load, environment, etc. The influence those factors have in structure topology optimization process cannot be ignored. (1)Variations in temperature. Actrually, the temperature is fluctuate, normally we see the fluctuation of temperature parameters as noise factor. (2)The change of raw material parameters. Raw material parameters usually be seen as controllable factor, but there is always a deviation between the actual value and the nominal value of raw material. The results of the topology optimization come out with certain differences as the geometry size is different. The factors like raw material parameters are usually be called as inner noise factors. (3)The load. Lord factor is the external load of products or process. Different loading have different deformation and stress on material. (4)Environment. Many production processes is related to environment conditions.

Experimental and Modeling Strategy. In the robust reliability optimal design, we explore the interaction of controllable factors and noise factors, thus we reduce the variable differences by changing the level combination of controllable factors. That is because the controllable factors are usually easily changed, so the robust reliability optimal design is more economical and convenient than reduce the noise difference directly.

Location and dispersion modeling try to build a model that its position and divergence corresponding to main effects and interaction effects. As for every combination of controllable level, use sample average of repeated noise trials as measure of location, use sample variance of logarithm $\ln s^{2}$ or sample variance $s^{2}$ as measure of divergence. For these two measures, we find out the factors which have remarkable impact on them respectively.

The Measurement of Position and Divergence. The measurement of position and divergence commonly use the mean and variance of sample, but the response variable optimization goals may is expected to be big,small and the order three kinds of form,Uniform signal-to-noise ratio and sensitivity is one of the taguchi recommendations.

Assume $y$ is looking at big features, $1 / y$ is looking at big features. Available at SNR estimation formula of looking at big features:

$$
\hat{\eta}=\frac{n}{\sum_{i=1}^{n} y_{i}^{-2}} .
$$

Take the logarithm multiplied by 10 , to a decibel value, then:

$$
\hat{\eta}=-10 \lg \frac{1}{n} \sum_{i=1}^{n} y_{i}^{-2}(d B) .
$$




\section{Case Study}

Considering the structure as shown in figure 1, outside the structure under applying a midpoint mechanical load as $F$, the Initial design domain is $80 \mathrm{~mm} \times 30 \mathrm{~mm}$, Solid material and the ratio of the whole design domain is $f$, Modulus of elasticity and Poisson's ratio are $E=100 G P_{a}, v=0.3$. Getting the maximum stiffness reliability optimal topology under the action of outer load and temperature load together. Among them, the $\Delta T$ can be set as $90 \mathrm{~K} 100 \mathrm{~K}$ and $110 \mathrm{~K}$, the $F$ can be set as $8 \mathrm{~N}, 10 \mathrm{~N}$, $12 \mathrm{~N}$, the $f$ can be set as $0.3,0.4,0.5$, the $\alpha$ can be set as $17.2 \times 10^{-6} / K, 18.2 \times 10^{-6} / \mathrm{K}, 19.2 \times 10^{-6} / \mathrm{K}$.

Using the MINITAB software for test design, for the error factors, set $\Delta T$ ( $\pm 10 \%), F$ ( $\pm 5 \%), f$ ( $\pm 5 \%)$ and $\alpha( \pm 10 \%)$. With the comprehensive error method, the collocation of various factors to select the most unfavorable situation of two test, the average value, signal-to-noise ratio and larger and smaller of strain energy as shown in table 1-3. The main effect of SNR is shown in Fig. 1. The average diagram of main effect is shown in Fig. 2.

Table 1 The data table of experimental simulation

\begin{tabular}{|c|c|c|c|c|c|c|c|c|}
\hline number & $\Delta T$ & $F$ & $f$ & $\alpha$ & $C_{1}$ & $C_{2}$ & $\eta_{1}$ & mean \\
\hline 1 & 90 & 8 & 0.3 & 17.2 & 359.881 & 359.037 & 51.1130 & 359.459 \\
\hline 2 & 90 & 10 & 0.4 & 18.2 & 360.324 & 359.004 & 51.1179 & 359.664 \\
\hline 3 & 90 & 12 & 0.5 & 19.2 & 360.179 & 359.075 & 51.1170 & 359.627 \\
\hline 4 & 100 & 8 & 0.4 & 19.2 & 362.784 & 362.137 & 51.1852 & 362.461 \\
\hline 5 & 100 & 10 & 0.5 & 17.2 & 363.842 & 363.026 & 51.2085 & 363.434 \\
\hline 6 & 100 & 12 & 0.3 & 18.2 & 365.163 & 364.983 & 51.2476 & 365.073 \\
\hline 7 & 110 & 8 & 0.5 & 18.2 & 365.548 & 365.038 & 51.2528 & 365.293 \\
\hline 8 & 110 & 10 & 0.3 & 19.2 & 366.682 & 366.184 & 51.2799 & 366.433 \\
\hline 9 & 110 & 12 & 0.4 & 17.2 & 367.149 & 366.887 & 51.2937 & 367.018 \\
\hline
\end{tabular}

Table 2 The response of Signal-to-noise ratio

\begin{tabular}{|c|c|c|c|c|}
\hline level & $\Delta T$ & $F$ & $f$ & $\alpha$ \\
\hline 1 & 51.12 & 51.18 & 51.21 & 51.21 \\
\hline 2 & 51.21 & 51.20 & 51.20 & 51.21 \\
\hline 3 & 51.28 & 51.22 & 51.19 & 51.19 \\
\hline Delta & 0.16 & 0.04 & 0.02 & 0.01 \\
\hline Rank & 1 & 2 & 3 & 4 \\
\hline
\end{tabular}

Table 3 The response of mean value

\begin{tabular}{|c|c|c|c|c|}
\hline level & $\Delta T$ & $F$ & $f$ & $\alpha$ \\
\hline 1 & 359.6 & 362.4 & 363.7 & 363.3 \\
\hline 2 & 363.7 & 363.2 & 363.0 & 363.3 \\
\hline 3 & 366.2 & 363.9 & 362.8 & 362.8 \\
\hline Delta & 6.7 & 1.5 & 0.9 & 0.5 \\
\hline Rank & 1 & 2 & 3 & 4 \\
\hline
\end{tabular}

In table 2, the most significant influence on the signal-to-noise ratio is the temperature. According to table 3, temperature is still the most significant impact factors, and next are the external load, volume ratio and thermal expansion coefficient. The best collocation of four factors: $\Delta T=110 \mathrm{~K}, F=12 \mathrm{~N}, f=0.3, \alpha=17.2 \times 10^{-6} / K$ or $\alpha=17.2 \times 10^{-6} / K$. In the process of topology optimization of TMC compliant mechanism, the $\Delta T$ is the largest influence factor, and $\alpha$ is smallest. So in the actual process, the influence of temperature should be focus on.

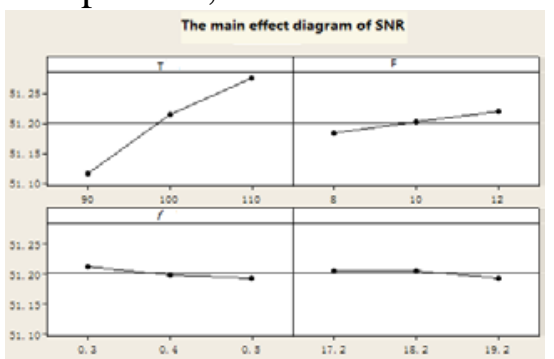

Fig. 1 The main effect of SNR

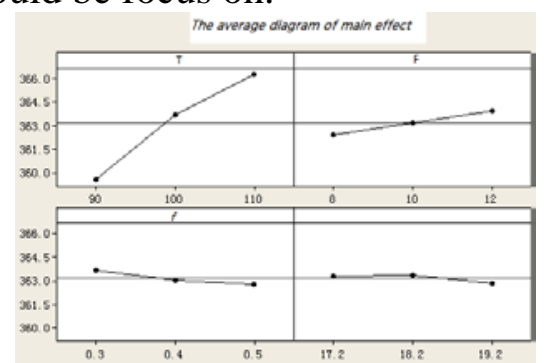

Fig.2 The average diagram of main effect

\section{Conclusion}

In combination with the robust reliability optimal design idea and topology optimization of TMC compliant mechanism, this paper gets the sensitivity of various influence factors on the topology optimization process. The differences between various influencing factors of differential structure 
which is formed by the tie-in way are obtained. The methods can be arranged by actual condition, with it the solid products can be obtained to adapt to the change of the influence factors.

\section{Acknowledgement}

This work was supported in part by Science and Technology Project of Jiangxi (GJJ12357).

\section{References}

[1] L.J. KANG and R.Q. LI: Machinery Design \& Manufacture, 2013, no.3, p. 7

[2] S.H. Ha, S. Cho: Numerical Heat Transfer, vol.48(2005), no.1, p.67

[3] A.L. Gain, G.H. Paulino: Structural and Multidisciplinary Optimization, vol.46(2012), p 327

[4] J.Q. ZHAN, X.M.ZHANG: Journal of Mechanical Engineering, vol.46(2010), no.13, p.42

[5] G. KHARMANDA, N. OLHOFF: Structural and Multidisciplinary Optimization, vol.26(2004), p. 295

[6] Z.K. LI, X.M. ZHANG: Journal of South China University of Technology, vol.36(2008), no.8, p.110

[7] Y.C. KUANG, G.F. OUYANG: Journal of Mechanical Strength, vol.31(2009), no.4, p.604

[8] M.T. CUI, J.J. CHEN, Y.Q.CHEN, et al: Journal of Xidian University, vol.34(2007),no.5,p.784

[9] D.M. LI, X.M. ZHANG, N.F. WANG, et al: Journal of South China University of Technology, vol.39 (2011), no.6, p.42

[10]D. LI, X. ZHANG, Y. GUAN, et al. IEEE/ASME International Conference on, 2010, p.403 